\title{
Tomato RNA-seq Data Mining Reveals the Taxonomic and Functional Diversity of Root-Associated Microbiota
}

\author{
Matteo Chialva ${ }^{1, *}{ }^{\circledR}$, Stefano Ghignone ${ }^{2}\left(\mathbb{D}\right.$, Mara Novero ${ }^{1}\left(\mathbb{D}\right.$, Wael N. Hozzein ${ }^{3,4}(\mathbb{D}$, \\ Luisa Lanfranco ${ }^{1}$ and Paola Bonfante ${ }^{1}$ \\ 1 Department of Life Sciences and Systems Biology, University of Torino, Viale P.A. Mattioli 25, \\ I-10125 Torino, Italy; mara.novero@unito.it (M.N.); luisa.lanfranco@unito.it (L.L.); \\ paola.bonfante@unito.it (P.B.) \\ 2 Institute for Sustainable Plant Protection, National Research Council of Italy (CNR), Viale P.A. Mattioli 25, \\ I-10125 Torino, Italy; stefano.ghignone@ipsp.cnr.it \\ 3 Bioproducts Research Chair, Zoology Department, College of Science, King Saud University, \\ 11451 Riyadh, Saudi Arabia; whozzein@ksu.edu.sa \\ 4 Botany and Microbiology Department, Faculty of Science, Beni-Suef University, Beni-Suef 62511, Egypt \\ * Correspondence: matteo.chialva@unito.it
}

Received: 2 December 2019; Accepted: 21 December 2019; Published: 24 December 2019

\begin{abstract}
Next-generation approaches have enabled researchers to deeply study the plant microbiota and to reveal how microbiota associated with plant roots has key effects on plant nutrition, disease resistance, and plant development. Although early "omics" experiments focused mainly on the species composition of microbial communities, new "meta-omics" approaches such as meta-transcriptomics provide hints about the functions of the microbes when interacting with their plant host. Here, we used an RNA-seq dataset previously generated for tomato (Solanum lycopersicum) plants growing on different native soils to test the hypothesis that host-targeted transcriptomics can detect the taxonomic and functional diversity of root microbiota. Even though the sequencing throughput for the microbial populations was limited, we were able to reconstruct the microbial communities and obtain an overview of their functional diversity. Comparisons of the host transcriptome and the meta-transcriptome suggested that the composition and the metabolic activities of the microbiota shape plant responses at the molecular level. Despite the limitations, mining available next-generation sequencing datasets can provide unexpected results and potential benefits for microbiota research.
\end{abstract}

Keywords: fungi; holobiont; meta-transcriptome; microbiota; RNA-seq; tomato

\section{Introduction}

"Omics" approaches have profoundly changed our views on environmental and host-associated microbiotas. Metagenomics has allowed researchers to explore the diversity and composition of fungal and bacterial communities under diverse environmental conditions [1], and meta-transcriptomics identifies the most relevant metabolic pathways operating in the microbiota. Such approaches have been applied to the human microbiota, linking microbial profiles with the emergence of specific diseases [2], and to plant microbiota, describing the metabolic processes of the microbes associated with different species of plants [3,4]. Moreover, because of the large amount of information generated by next-generation sequencing (NGS), plant RNA-seq datasets that were originally generated to study the host transcriptome may be a novel resource for studying the plant-associated microbiota [5]. 
Some studies have focused on host-microbe pairs under controlled laboratory conditions, in so-called "dual RNA-seq" approaches and applied to plants such as cassava (Manihot esculenta) colonized by different arbuscular mycorrhizal (AM) fungal isolates [6] and Norway spruce (Picea abies) infected with Heterobasidion annosum [7]. Plant RNA-seq datasets have also been used to study the plant-associated microbiota, for example in the perennial grass Holcus lanatus growing in different soils [8]. These approaches are often limited by sequencing depth or read length, requiring assembly and annotation of the reads [5,9], and may have potential drawbacks such as the assembly of chimeric transcripts [10]. They demonstrate, however, how unbiased RNA-sequencing may reveal not only the biochemical functions being performed by a complex microbial community, but also the species within the community that are metabolically active.

Here, we used an RNA-seq dataset previously generated for tomato plants (Solanum lycopersicum) growing on different native soils [11,12] to test the hypothesis that host-targeted transcriptomics can detect the taxonomic and functional diversity of the root microbiota. We also examined potential correlations of microbiome diversity and activity with specific plant gene expression profiles. The previous research, which integrated transcriptomics and proteomics, was focused on the plant side-it revealed that two tomato cultivars, growing in native soils with different biological properties, responded to natural soil microbiotas by increasing their innate immunity and activating basal defense responses. The transcriptomic response of the plants depended more on soil type, and likely on the microbiota present, than on plant genotype. Parallel investigations of the cultivable fungi and of the tomato-associated mycobiota using internal transcribed spacer (ITS)-metabarcoding revealed consistent differences in the fungal community assemblage depending on the soil and plant genotype $[13,14]$. Here, we focus our attention on the microbial side, covering both fungal and bacterial communities.

To verify the feasibility of our approach, we developed a bioinformatic pipeline that was able to taxonomically and functionally annotate short RNA-seq reads. The pipeline takes advantage of read alignment, lowest common ancestor assignments, and functional annotation to reconstruct the diversity of active microbes associated with the roots of the two tomato cultivars grown on different native soils or on a control substrate. Our analysis succeeded in rendering bacterial and fungal diversity profiles, as well as identifying active functional categories. Applying the pipeline to our system we verified the hypothesis that microbial functions are also impacted by plant pathogen tolerance and soil suppressiveness. Finally, using genome-wide RNA-seq data mapped on tomato we further correlated the diversity and functional profiles to the host transcriptome.

\section{Materials and Methods}

\subsection{Plant Material and Sequencing}

The RNA-seq libraries analyzed in this study were obtained in a previous study [11]. The experimental setup consisted of two tomato (Solanum lycopersicum) genotypes, "Battito" (B) and "Cuore Di Bue" (C), which are resistant and susceptible, respectively, to Fusarium oxysporum f.sp. lycopersici (FOL) pathogen (races 1 and 2). Both genotypes were grown in a greenhouse pot experiment in two field-sampled soils, "Albenga" (AL) and "Rosta" (RO), which are suppressive and conducive, respectively, to FOL and in a disinfected peat-based substrate as a control (CONT). For each condition, three biological replicates were considered. A complete characterization of soil physicochemical features is available in Poli et al. [13]. Details of the sampling protocol, RNA extraction, and Illumina sequencing (50 bp single-end) procedures are described in Chialva et al. [11]. Raw RNA-seq reads are available in the NCBI Short Read Archive repository [15] under accession no. SRP126554. The same dataset was used to analyze the tomato transcriptome and to reconstruct the microbial diversity associated with plant roots as described below. 


\subsection{Plant Transcriptome Analysis}

Raw RNA-seq libraries were analyzed according to the bioinformatic pipeline described in Chialva et al. [12], using the Heinz tomato genome (SL2.4) as the reference [16] and normalizing expression values with the DESeq2 package [17] in R [18].

\subsection{Meta-Transcriptome Filtering and Annotation}

Reads originating from microbial populations were filtered and annotated using a custom bioinformatic pipeline. Because the short read length could lead to the assembly of chimeric sequences, we chose to annotate reads rather than generating an assembly.

Raw adapter-filtered reads were first trimmed using TRIMMOMATIC v0.35 [19] setting Q $>28$ (with a 10-base sliding window) and length $>45 \mathrm{bp}$. Host reads were then removed by mapping trimmed libraries on the tomato reference genome (SL.2.50) [16] using STAR c2.2.5a [20] in "end-to-end" mode, allowing a maximum number of 1000 multi-reads and setting intron length limits to 40 and 23,000 bp. To remove residual host reads, accounting for transcript variants and possible contaminants, we mapped reads on a NCBI NT subset containing all tomato and human sequences using Bowtie2 v2.2.9 [21] in "end-to-end" mode with "-D $25-\mathrm{R} 4$-N 1 -L 15 -i S,1,0.50" parameters. The rRNA sequences were then removed using Sortmerna v2.1 [22], using "-L 14 -passes 14,7,1 -e 1" parameters and the built-in databases plus a comprehensive subset of tomato rRNAs from the NCBI NT database [23] as the reference.

Filtered reads were then annotated on the whole NCBI NT database (release 13 July 2016) using the lowest common ancestor method (LCA) implemented by Taxoner64 v1.7 [24] according to the complete NCBI taxonomy (release 13 July 2016). Taxoner64 was run using the previously used Bowtie 2 parameters (see above) and assigning reads to the nearest neighbor taxon ID at $97 \%$ sequence similarity.

Output files, containing reads annotated by taxid and GI (now accession number), were further processed in $\mathrm{R}$ [18] with custom scripts to obtain read counts per taxa. Taxonomic annotation for each taxon was retrieved using the CHNOSZ library [25], and data were further filtered to remove residual contaminants (Viridiplantae and Metazoa) as well as low-abundance taxa $(<5$ raw reads in at least 3 libraries).

As a further step, we functionally annotated the filtered microbial reads from the previous analysis by mapping to the eggNOG database v4.5 [26]. To overcome the short read length (50 bp), which did not allow functional assignment using conventional tools (such as MG-RAST or BLASTX), we generated a set of "pseudo-reads" extending reads $50 \mathrm{bp}$ upstream and $50 \mathrm{bp}$ downstream on their mapping reference in NCBI, thus generating longer $150 \mathrm{bp}$ fragments. Pseudo-reads were generated taking into account reference sequence start and end coordinates and extracting selected ranges (150 bp) using the "getfasta" function in Bedtools v2.26.0-19 [27]. Pseudo-reads from different samples were then pooled and mapped on eggNOG using DIAMOND-BLASTX v0.8.24 [28] with -k1 -e0.001 -b15 -c1 parameters and removing redundant high-scoring segment pairs (HSPs).

\subsection{Analysis of Arbuscular Mycorrhizal Colonization}

Mycorrhizal colonization was assessed on a representative portion of the same root materials used to generate RNA-seq libraries. Immediately after sampling, roots were washed thoroughly under flowing tap water, stained for at least $12 \mathrm{~h}$ in methyl blue $(0.1 \% w / v$ in lactic acid), and then bleached in lactic acid ( 3 washes, $1 \mathrm{~h}$ each). Root samples were then cut into $1 \mathrm{~cm}$ segments, mounted on glass slides, and observed by light microscopy. Mycorrhizal colonization parameters were visually estimated according to Trouvelot et al. [29]. For each condition, 9 different plants were analyzed, with 60 root segments examined for each plant. 


\subsection{Statistical Analysis}

Statistical tests were performed in the R statistical programming environment [18]. Normalized read counts were grouped at the family level for bacteria and fungi. Permutational analysis of variance (PERMANOVA) of bacterial and fungal communities were computed using the "vegan" package v2.5-6 in R [30].

Differential abundance analysis was performed using the DESeq2 package v1.12.14 [17] to increase sensitivity relative to traditional rarefaction techniques [31]. The DESeq2 function was run using the independent filtering option and setting fitType $=$ "parametric" and betaPrior $=\mathrm{T}$. Taxa were considered as differentially abundant between conditions at an adjusted $p$-value $<0.05$.

Statistical and differential Clusters of Orthologous Groups (COG) abundance analyses were obtained as previously described for taxa. Non-metric multidimensional scaling (NMDS) ordination analysis on functional categories was performed using "vegan" R package v2.5-6 [31]. Additionally,

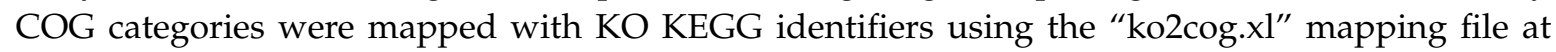
http://www.genome.jp/kegg/files/ko2cog.xl. Enriched KEGG pathways among KO IDs were then inferred using the clusterProfiler v3.14.0 package in R [32] at $p<0.1$.

Data normality and homoscedasticity were tested using Shapiro-Wilk [33] and Levene's test [34] in the "stats" v3.6.1 and "car" v3.0-3 packages [34], respectively $(p<0.05)$. According to data distributions, ANOVA was adopted for normal homoscedastic data and the Kruskal-Wallis test adopted for non-normal homoscedastic data [35] from the custom R package "stats" v3.6.1 at $p<0.05$. Pairwise comparisons between treatments were performed when needed, using the appropriate post hoc tests. Tukey's test [36] in the package "agricolae" v1.3-1 [37] was adopted for ANOVA, and Dunn's test [38] in package "FSA" v0.8.25 [39] for Kruskall-Wallis, both at $p<0.05$.

Variance partitioning analyses were used to explain plant transcriptome variance, using as explanatory variables meta-transcriptome diversity (fungi and bacteria) and functional diversity, as well as genotype and soil factors. DESeq2-normalized counts for microbial taxa and COG categories were used as meta-transcriptome descriptors. The analyses were performed using the "varpar" function in the "vegan" package [31]. Since collinearity was detected within datasets, a forward-selection procedure [40] was applied using the "forward.sel" function in the "adespatial" package v0.3-7 [41] with alpha $=0.05$ and nperm $=999$. Testable partitions were tested for significance using permutational ANOVA (999 permutation) on the RDA model $(p<0.05)$.

All analyses were performed on a HP Proliant server (64 cores, 128 GB RAM) running Ubuntu server 14.04. Custom $\mathrm{R}$ functions used to perform analyses are available at https://github.com/mchialva.

\section{Results and Discussion}

\subsection{Reconstructing the Root-Associated Meta-Transcriptome from Host-Targeted RNA-seq Libraries}

The RNA-seq dataset analyzed here was obtained from roots of two tomato genotypes grown in two native soils and one inert, control substrate in pots [11]. By observing the RNA-seq mapping rate onto the tomato reference genome, we noticed that a variable proportion of reads, ranging from $4 \%$ to $25 \%$, were not aligning to the host genome. To uncover the diversity of active species in the microbial communities associated with tomato roots and their functional activities, we implemented a custom bioinformatic pipeline to filter reads generated by microbial transcripts (meta-transcriptome). The pipeline was composed of several steps, including reads trimming and filtering procedures, host reads and rRNA removal, and meta-transcriptome annotation (Figure S1). Taxonomic affiliations and functional annotations were obtained using the full NCBI Nucleotide database [23] and the eggNOG database [26], respectively.

Our pipeline detected from 93,736 to 581,439 microbial reads, depending on the sample, within the filtered sequences (Table 1). The vast majority of sequences ( $81 \%$ to $96 \%$, depending on the library) were unique, displaying low redundancy. The mapping rate on the NCBI database ranged between $8.59 \%$ and $42.85 \%$ (Table 1), representing a variable proportion of bacteria and eukaryotes (Figure 1a). 
Table 1. Meta-transcriptome reads assignment statistics.

\begin{tabular}{|c|c|c|c|c|c|c|c|c|c|}
\hline Sample & SRA $^{1}$ Run & $\begin{array}{c}\text { SRA }^{1} \text { Sample } \\
\text { Accession }\end{array}$ & Raw & Filtered & $\begin{array}{c}\text { Unique Filtered } \\
\text { Reads (\%) }\end{array}$ & $\begin{array}{c}\text { Mapped on } \\
\text { NCBI (\%) }\end{array}$ & Bacteria & Eukaryotes & $\begin{array}{l}\text { Mapped \% } \\
\text { (eggNOG) }\end{array}$ \\
\hline BAL_R1 & SRR6368019 & SRS2751529 & $11,382,297$ & 184,182 & 88.88 & 27.12 & 4952 & 16,074 & 33.41 \\
\hline BAL_R2 & SRR6368032 & SRS2751512 & $20,308,970$ & 391,019 & 93.74 & 25.88 & 8662 & 34,437 & 34.28 \\
\hline BAL_R3 & SRR6368031 & SRS2751523 & $12,614,238$ & 187,812 & 95.77 & 41.37 & 3141 & 54,951 & 59.10 \\
\hline BRO_R1 & SRR6368030 & SRS2751514 & $14,986,370$ & 230,155 & 95.61 & 15.59 & 2909 & 7937 & 23.69 \\
\hline BRO_R2 & SRR6368029 & SRS2751516 & $15,367,923$ & 207,560 & 95.43 & 17.80 & 2853 & 8265 & 18.92 \\
\hline BRO_R3 & SRR6368036 & SRS2751513 & $17,906,164$ & 484,731 & 95.56 & 10.99 & 5185 & 13,803 & 29.70 \\
\hline BCONT_R1 & SRR6368035 & SRS2751519 & $12,893,929$ & 93,736 & 85.68 & 19.14 & 5114 & 676 & 29.46 \\
\hline BCONT_R2 & SRR6368034 & SRS2751517 & $23,428,531$ & 382,171 & 84.86 & 42.85 & 4751 & 98,337 & 44.00 \\
\hline BCONT_R3 & SRR6368033 & SRS2751518 & $13,161,547$ & 117,290 & 91.33 & 34.91 & 2982 & 8861 & 23.11 \\
\hline CAL_R1 & SRR6368022 & SRS2751525 & $13,376,685$ & 342,990 & 88.63 & 8.59 & 4691 & 7049 & 36.23 \\
\hline CAL_R2 & SRR6368021 & SRS2751526 & $12,343,880$ & 317,136 & 81.39 & 13.11 & 9361 & 7628 & 34.98 \\
\hline CAL_R3 & SRR6368024 & SRS2751522 & $18,637,725$ & 380,329 & 89.13 & 9.16 & 5928 & 6830 & 32.38 \\
\hline CRO_R1 & SRR6368023 & SRS2751524 & $13,977,334$ & 208,810 & 90.82 & 10.80 & 4651 & 3801 & 31.17 \\
\hline CRO_R2 & SRR6368026 & SRS2751530 & $18,315,136$ & 534,056 & 89.60 & 10.61 & 9998 & 13,518 & 34.78 \\
\hline CRO_R3 & SRR6368025 & SRS2751520 & $27,710,101$ & 581,439 & 91.36 & 12.77 & 8804 & 8195 & 18.68 \\
\hline CCONT_R1 & SRR6368028 & SRS2751515 & $17,006,711$ & 138,440 & 89.62 & 17.90 & 3071 & 820 & 13.90 \\
\hline CCONT_R2 & SRR6368027 & SRS2751521 & $29,267,531$ & 246,552 & 92.28 & 22.81 & 11,392 & 2488 & 22.10 \\
\hline CCONT_R3 & SRR6368020 & SRS2751527 & $16,244,284$ & 98,253 & 94.61 & 16.58 & 2804 & 1210 & 21.72 \\
\hline
\end{tabular}

${ }^{1}$ Short Read Archive respository at NCBI, https://www.ncbi.nlm.nih.gov/sra. 
a

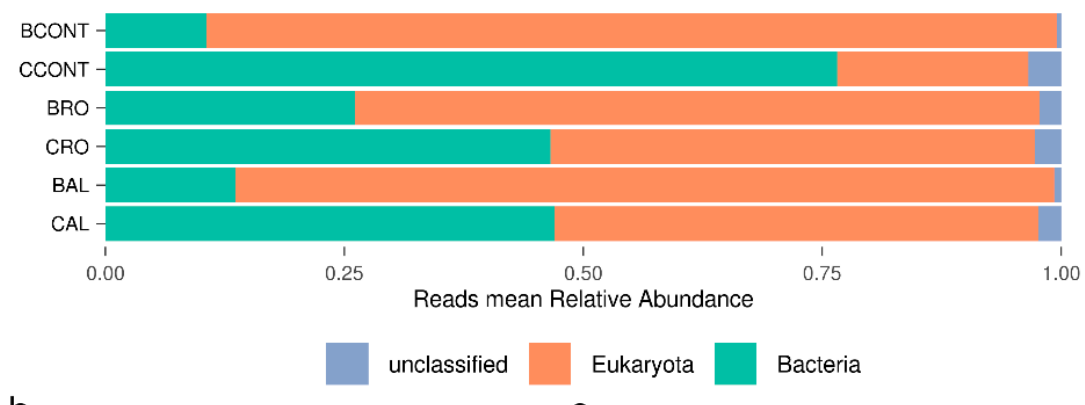

b

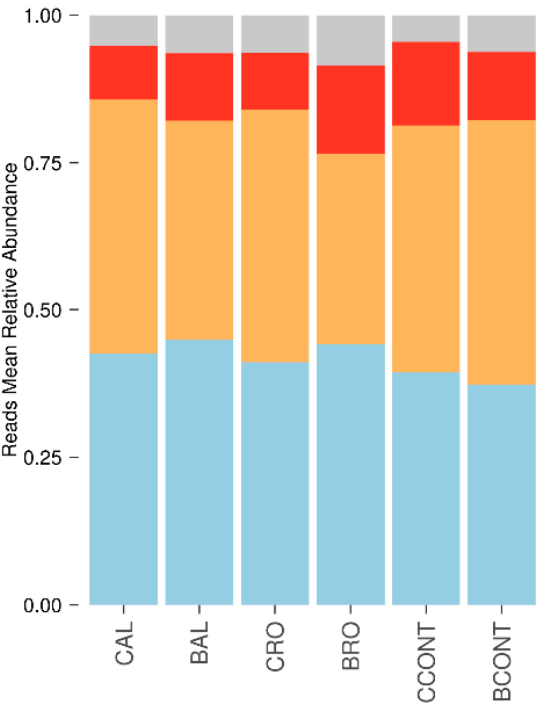

Fungi

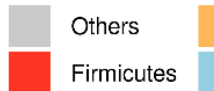

Proteobacteria

Actinobacteria
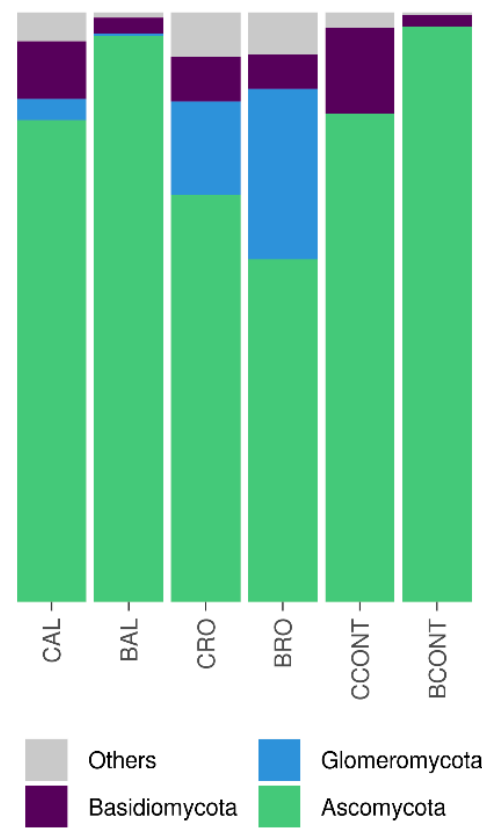

Figure 1. Active taxonomic diversity of root-associated microbiota of tomato plants grown in native and control soils. (a) Relative abundance of reads in each condition by superkingdom. (b,c) Bar plots of the three most represented phyla for bacteria (b) and fungi (c) among the different conditions. The first letter of the sample name indicates genotype ("Cuore di Bue" or "Battito"), while the following part refers to the soil/substrate type (RO, "Rosta" conducive soil; AL, "Albenga" suppressive soil; control (CONT), neutral peat-moss substrate).

Even though the Illumina libraries were enriched in poly-A transcripts, because they were initially targeted to the host plant, the analysis was successful in detecting a significant number of bacterial reads. The bacterial read counts were similar to those corresponding to fungi, possibly due to the presence of poly-A stretches in the bacterial transcripts. As frequently reported in similar studies, only a small proportion of the non-host reads could be successfully assigned [42,43], and in our case, this was probably due to the short read length $(50 \mathrm{bp} \mathrm{SE})$. Since the sampling protocol used to generate the RNA-seq dataset included repeated $\mathrm{dH}_{2} \mathrm{O}$ washes to remove most of the rhizospheric microbes [11], we hypothesized that the microbial meta-transcriptome reads could be attributed to both the rhizoplane and the endosphere root compartments. For this reason, we refer to these sequences as belonging to root-associated communities throughout. Indeed, all of the fungal and bacterial families we detected (see below) are well-known to be tightly associated with plant roots [44-47] and belong to diverse ecological guilds, from symbionts to saprotrophs.

\subsection{Tomato Root-Associated Active Microbiota Diversity Is Shaped by Both Soil Type and Host Genotype}

Most of the bacterial reads were assigned to Actinobacteria and Proteobacteria, while the fungal reads were dominated by Ascomycota (Figure 1b,c). At the phylum level (Figure 1b,c), a similar overall 
bacterial community composition emerged, with major variation across conditions observed only for fungi.

Indeed, the relative abundance of Glomeromycotina (Glomeromycota according to NCBI taxonomy) seemed to be dependent on soil type; this taxon was detected in both native soils but not in the control substrate.

As expected, the analysis showed that root-associated communities also were present in plants growing on the disinfected substrate, demonstrating quick re-colonization of a microbe-depleted environment in the non-sterile greenhouse conditions used for these experiments.

The taxa-count tables we obtained for fungi and bacteria were then used to investigate the effect of soil versus plant genotype on microbial diversity (at the phylum and family levels). The PERMANOVA showed that host genotype explained a large part of the variance in community composition for bacteria $(20.10 \%$ of variance explained, $p<0.01)$ and fungi $(28.83 \%, p<0.001)$ at the family level (Table 2). By contrast, soil type explained $20.25 \%$ of the variance $(p<0.05)$ only for the fungal community. These results are in agreement with data reported by Poli et al. [13] showing that the fungal communities tightly associated with tomato roots appeared to be mainly shaped by plant genotype.

We performed a differential abundance analysis comparing the "Cuore di Bue and "Battito" cultivars (Table S1).

Table 2. PERMANOVA analysis of meta-transcriptome diversity and functioning in relation to host genotype, soil, and its interaction. Df = degrees of freedom; SS = sum of squares; MS = mean sum of squares; Pseudo-F = F value by permutation. Statistical significance is indicated in bold $(p<0.05)$; $p$-values are based on 999 permutations.

\begin{tabular}{|c|c|c|c|c|c|c|c|}
\hline Source & Df & SS & MS & $\mathbf{F}$ & $R^{2}$ & $p$ & Explained Variance (\%) \\
\hline \multicolumn{8}{|c|}{ Bacteria (family) } \\
\hline Genotype & 1 & 0.10739 & 0.107388 & 4.5390 & 0.20102 & 0.0032 & 20.10 \\
\hline Soil & 2 & 0.05908 & 0.029542 & 1.2487 & 0.11060 & 0.2511 & 11.06 \\
\hline Genotype $\times$ Soil & 2 & 0.0838 & 0.041915 & 1.7716 & 0.15692 & 0.0762 & 15.69 \\
\hline Residual & 12 & 0.28391 & 0.023659 & & 0.53145 & & 53.15 \\
\hline Total & 17 & 0.53421 & & & 1 & & 100 \\
\hline \multicolumn{8}{|c|}{ Fungi (family) } \\
\hline Genotype & 1 & 0.69477 & 0.69477 & 7.6321 & 0.28835 & 0.0004 & 28.83 \\
\hline Soil & 2 & 0.48786 & 0.24393 & 2.6796 & 0.20247 & 0.0294 & 20.25 \\
\hline Genotype $\times$ Soil & 2 & 0.13449 & 0.06724 & 0.7387 & 0.05581 & 0.6368 & 5.58 \\
\hline Residual & 12 & 1.09239 & 0.09103 & & 0.45337 & & 45.34 \\
\hline Total & 17 & 2.40951 & & & 1 & & 100 \\
\hline \multicolumn{8}{|c|}{ COG genes } \\
\hline Genotype & 1 & 0.3782 & 0.3781 & 2.7336 & 0.11915 & 0.0253 & 11.92 \\
\hline Soil & 2 & 0.7818 & 0.39089 & 2.8254 & 0.24630 & 0.0091 & 24.63 \\
\hline Genotype $\times$ Soil & 2 & 0.3539 & 0.17696 & 1.2791 & 0.11151 & 0.2334 & 11.15 \\
\hline Residual & 12 & 1.6601 & 0.13835 & & 0.52304 & & 52.30 \\
\hline Total & 17 & 3.1740 & & & 1 & & 100 \\
\hline
\end{tabular}

Some Actinobacteria and Proteobacteria families that are commonly associated with the root endosphere and rhizosphere [48,49] were enriched or depleted in "Cuore di Bue" compared with "Battito" cultivars (Figure 2a). These families included Brucellaceae $\left(\log _{2} \mathrm{FC}=1.48\right)$, Methylobacteriaceae $\left(\log _{2} \mathrm{FC}=1.45\right)$, Burkholderiaceae $\left(\log _{2} \mathrm{FC}=1.07\right)$, Pasteurellaceae $\left(\log _{2} \mathrm{FC}=-1.23\right)$, Alcaligenaceae $\left(\log _{2} \mathrm{FC}=-1.27\right)$, Corynebacteriaceae $\left(\log _{2} \mathrm{FC}=-0.84\right)$, and Micrococcaceae $\left(\log _{2} \mathrm{FC}=-0.48\right)$. Among the fungi, the susceptible cultivar "Cuore di Bue" hosted fewer Nectriaceae (Ascomycota), while other Ascomycota members, such as Mycospherellaceae, Metschnikowiaceae and Chytridiomycota were enriched (Figure $2 b$ ). Comparison of the two genotypes revealed a higher abundance in the resistant cultivar "Battito" of some fungal taxa such as Nectriaceae, which includes many Fusarium strains that 
can be plant pathogens or biocontrol agents. This result suggests that the resistant genotype readily recruits biocontrol Fusaria strains, which can putatively confer resistance against FOL as reported in the literature [50].
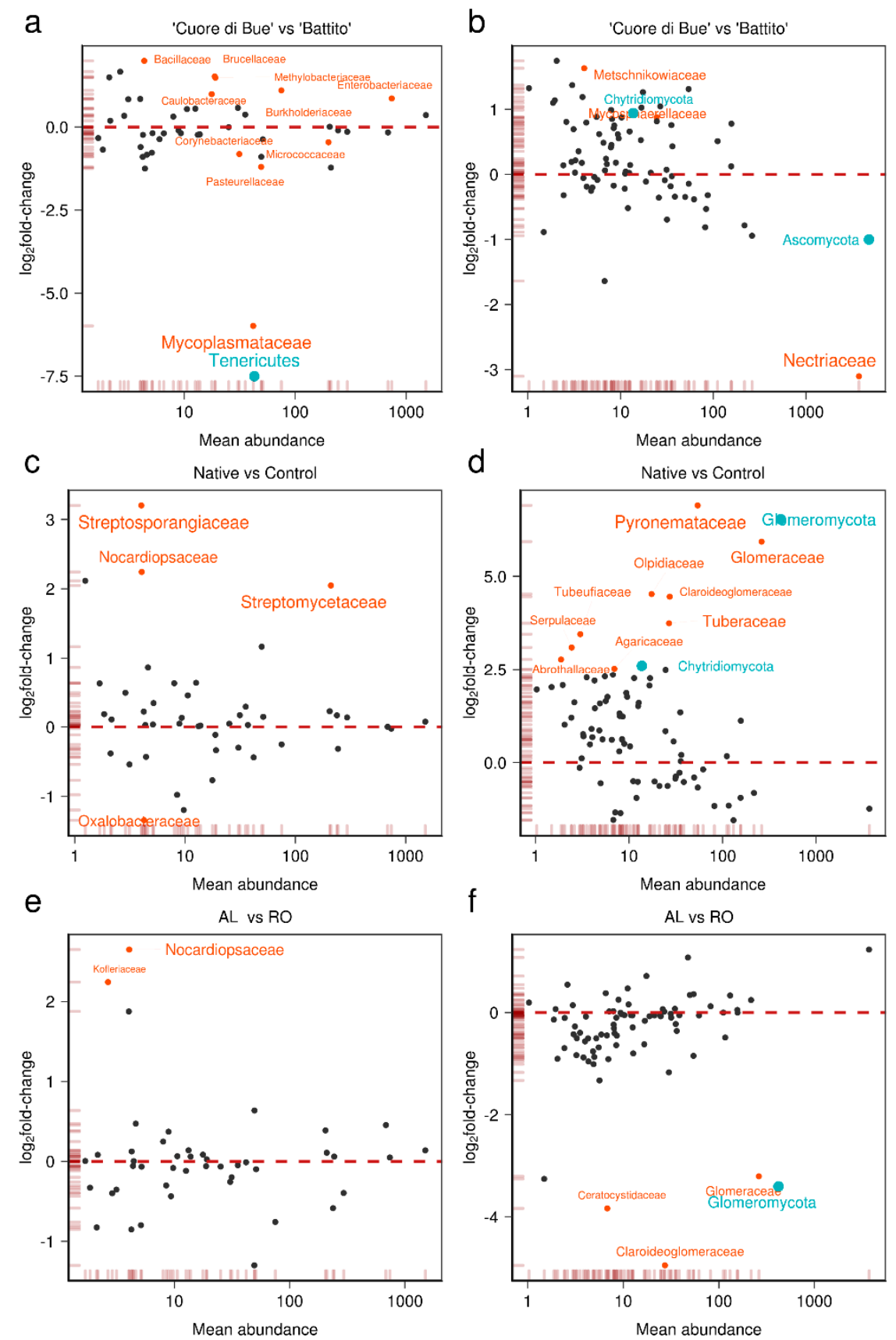

Figure 2. MA-plots of differential abundance analysis of bacterial and fungal families/phyla across genotype and soil types. (a,b) Enriched and depleted taxa of bacteria (a) and fungi (b) associated with "Cuore di Bue" versus "Battito" cultivars. (c,d) Enriched and depleted families of bacteria (c) and fungi (d) in native versus control soil. (e,f) Enriched and depleted families of bacteria (e) and fungi (f) in disease suppressive versus conducive soils. Differentially expressed (False Discovery Rate [FDR] $<0.05)$ families and phyla are depicted in pale red and light blue, respectively. Taxa name sizes are $\log _{10}$-proportional to the adjusted $p$-value (FDR). Rug density plots (pale red) along each axis indicate the density of abundances ( $x$-axis) and $\log _{2}$ fold-changes ( $y$-axis); $x$-axis is $\log _{10}$-scaled. 
We also tested which taxa were enriched in native soils versus the neutral control. The results highlighted four bacterial families and 10 fungal families as differentially abundant in native soils (Table S1).

Among bacteria, Actinobacteria such as Streptosporangiaceae $\left(\log _{2} \mathrm{FC}=3.19\right)$, Nocardiopsaceae $\left(\log _{2} \mathrm{FC}=2.23\right)$, and Streptomycetaceae $\left(\log _{2} \mathrm{FC}=2.04\right)$ emerged as predominant components of the native microbiota (Figure 2c). Among fungi (Figure 2d), Glomeraceae $\left(\log _{2} \mathrm{FC}=5.97\right.$ ), Claroideoglomeraceae $\left(\log _{2} \mathrm{FC}=4.46\right)$, Tuberaceae $\left(\log _{2} \mathrm{FC}=3.77\right)$ and Pyronemataceae $\left(\log _{2} \mathrm{FC}=6.89\right)$ were enriched in native soils relative to the control.

Compared to native soils, the control soil samples had a greater abundance of the bacterial family Oxalobacteraceae $\left(\log _{2} \mathrm{FC}=-1.34\right.$ ) (Figure $2 \mathrm{c}$ ) but were not enriched for any fungal taxa (Figure 2c,d). Interestingly, Oxalobacteraceae, which is commonly found in soil and in association with the root endosphere, was previously described as one of the most persistent bacterial taxa associated with cucumber seeds [51]. If Oxalobacteraceae are similarly persistent in their association with tomato seeds, they might be the first to re-colonize the disinfected substrate (CONT), since no competition with any other microbes would exist.

The type of soil also influenced the root-associated microbiota. Specifically, we observed an enrichment of the bacterial families Nocardiopsaceae and Kofleriaceae in AL suppressive soil (Figure 2e). Interestingly, these two families were previously associated with soil disease suppressiveness because of their anti-fungal activity [52], confirming the disease-suppressive potential of AL soil.

Among fungi (Figure 2f), arbuscular mycorrhizal (AM) fungi (Glomeraceae and Claroideoglomeraceae) as well as pathogenic fungi such as Ceratocystidaceae were less abundant in the AL disease-suppressive soil (False Discovery Rate [FDR] < 0.05).

Funneliformis mosseae was the most represented AM fungus. Interestingly, this species is cosmopolitan, able to colonize roots of a large number of different plants and is highly resistant to soil disturbances $[53,54]$, since AM fungi are easily detectable by light microscopy after root staining, as we confirmed in the molecular results by examining root segments (Figure 3 ).

Quantitative analysis revealed a significant difference $(p<0.05)$ between RO and AL soils (Figure 3a), with a lower occurrence of fungal structures and arbuscules in roots of both tomato genotypes grown in AL suppressive soil. The decreased presence of AM fungi in the suppressive soil AL, along with a higher abundance of bacteria with well-known anti-fungal activities, raises novel questions about the fungal dynamics in suppressive soils [55].

\subsection{Basal Microbial Metabolisms Are Detected in the Reconstructed Meta-Transcriptome}

Reads assigned by the pipeline to bacterial and fungal taxa were pooled and functionally annotated to dissect microbial functional diversity associated with tomato roots under different soil and genotype conditions (Table S2). Reads were annotated using the eggNOG functional database [26] and differential expression analysis of Clusters of Orthologous Groups (COG) genes was performed. The mean mapping rate ranged between $13.9 \%$ and $59.10 \%$ of filtered reads (Table 1 ).

PERMANOVA analysis on COGs profiles (Table 2) showed that the largest part of the variation was linked to the soil type ( $p<0.01,24.63 \%$ explained variance), but also that the genotype has a relevant role ( $p<0.05,11.91 \%$ explained variance).

Distances between functional profiles were visualized through non-metric multidimensional scaling (NMDS) ordination analysis, clustering genotypes, and soil type (Figure $4 \mathrm{a}, \mathrm{c}$ ). The ordination was well-supported by PERMANOVA analysis, which detected an effect of genotype and soil. The plot revealed a different pattern between the two tomato genotypes, with a clear separation between them (Figure 4a). Similarly, soil types also clustered apart, with a large overlap between the two native soils and a clear separation of the control substrate (Figure 4c). 

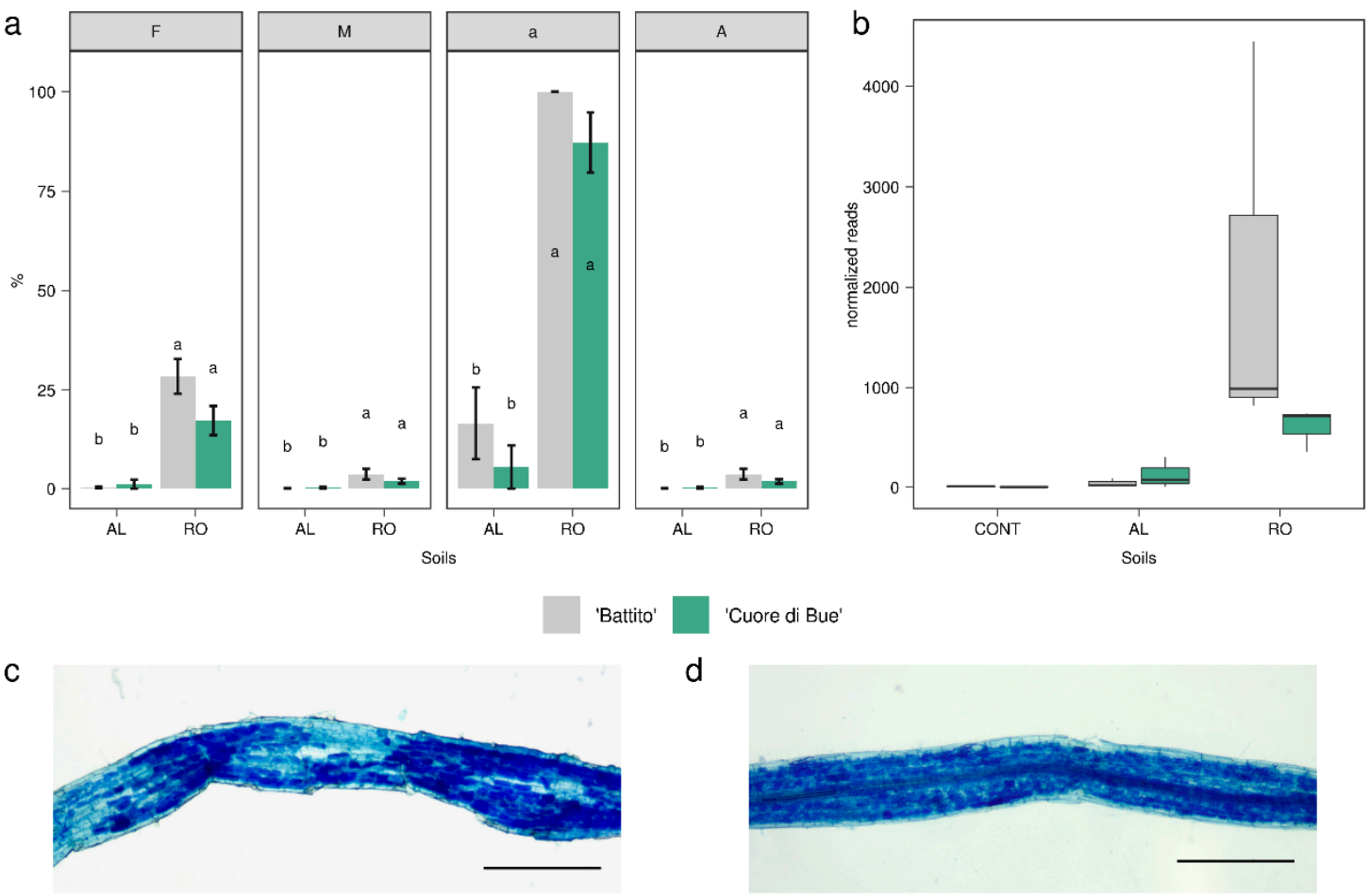

'Cuore di Bue'

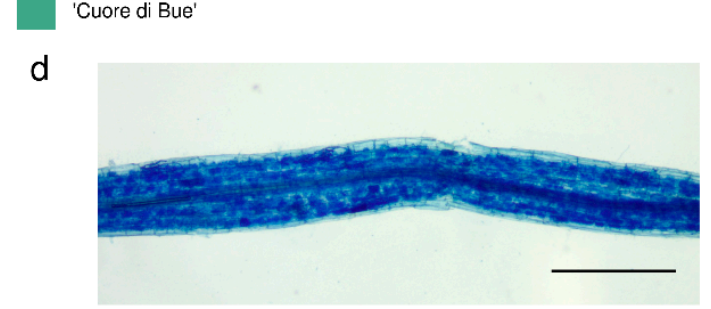

Figure 3. Mycorrhizal colonization of tomato roots growing in native soils and arbuscular mycorrhizal fungi (AMF) reads abundance. (a) Quantitative parameters of arbuscular mycorrhizal (AM) colonization of tomato roots (susceptible and resistant genotypes) growing in two different native soils: Mycorrhizal frequency $(\mathrm{F})$, intensity of mycorrhization $(\mathrm{M})$, presence of arbuscules within the mycorrhized segments (a), and the presence of arbuscules in the whole root apparatus (A) are plotted. Letters indicate significant differences among means according to the Kruskall-Wallis test (Dunn's post hoc test, $p<0.05$ ). (b) Normalized reads relative abundances assigned to phylum Glomeromycotina in the meta-transcriptome analysis. (c,d). Micrographs showing AMF colonization in tomato roots from AL (c) and RO (d) natural soils. Scale bars correspond to $30 \mu \mathrm{m}$ in (e) and to $25 \mu \mathrm{m}$ in (f).

We next compared expression of COGs in susceptible ("Cuore di Bue") versus resistant ("Battito") cultivars (Table S2) and found 210 differentially expressed COGs, with 33 up-regulated and 177 down-regulated (FDR < 0.05) genes (Figure $4 b$ ). Interestingly, with few exceptions, most of the differentially expressed COGs were depleted in the susceptible cultivar "Cuore di Bue" (i.e., they were enriched in the resistant "Battito" cultivar). Differentially expressed genes mainly belonged to a few functional categories: "Function unknown (S)" (76 COGs); "Carbohydrate transport and metabolism (G)" (20 COGs); "Posttranslational modification, protein turnover, chaperones (O)" (20 COGs); "Intracellular trafficking, secretion, and vesicular transport (U)" (12 COGs); “Translation, ribosomal structure and biogenesis (J)" (12 COGs); "Signal transduction mechanisms (T)" (11 COGs); and "Amino acid transport and metabolism (E)" (10 COGs). Among the most interesting of the down-regulated gene categories we found in the "Cuore di Bue" cultivar were calcium calmodulin-dependent protein kinases (ENOG410XRMJ, ENOG410XNRX) and serine threonine protein kinases (COG0515, ENOG410XNPH), both of which are categories involved in signal transduction.

When we analyzed native soils versus control, we found 49 differentially expressed COGs, with a similar number of up- and down-regulated categories (Figure 4d). Up-regulated COGs mostly belonged to the "Translation, ribosomal structure and biogenesis (J)" category (15/24 COGs). Down-regulated genes were assigned to a few other categories, such as "Energy production and conversion $(\mathrm{C})$ ", "Function unknown (S)", and "Carbohydrate transport and metabolism $(\mathrm{G})$ ", with eight, six, and four COGs terms, respectively. Of the 15 upregulated COGs assigned to category " $\mathrm{J}$ ", five corresponded to ribosomal proteins (COG1717, COG1997, COG1631, COG1632, COG1358). Since ribosomal proteins 
have been proposed as markers of in situ growth rates in microbial communities [56], our data suggest that in native soils, as compared to the control substrate, the higher microbial abundance associated with plant roots is mirrored by a higher microbial metabolic activity. Despite the differences between the native soils and the control, we found no COGs that were differentially expressed between the suppressive and conducive soils. This supports the NMDS ordination analysis, which displayed high similarities between AL and RO COGs expression profiles.

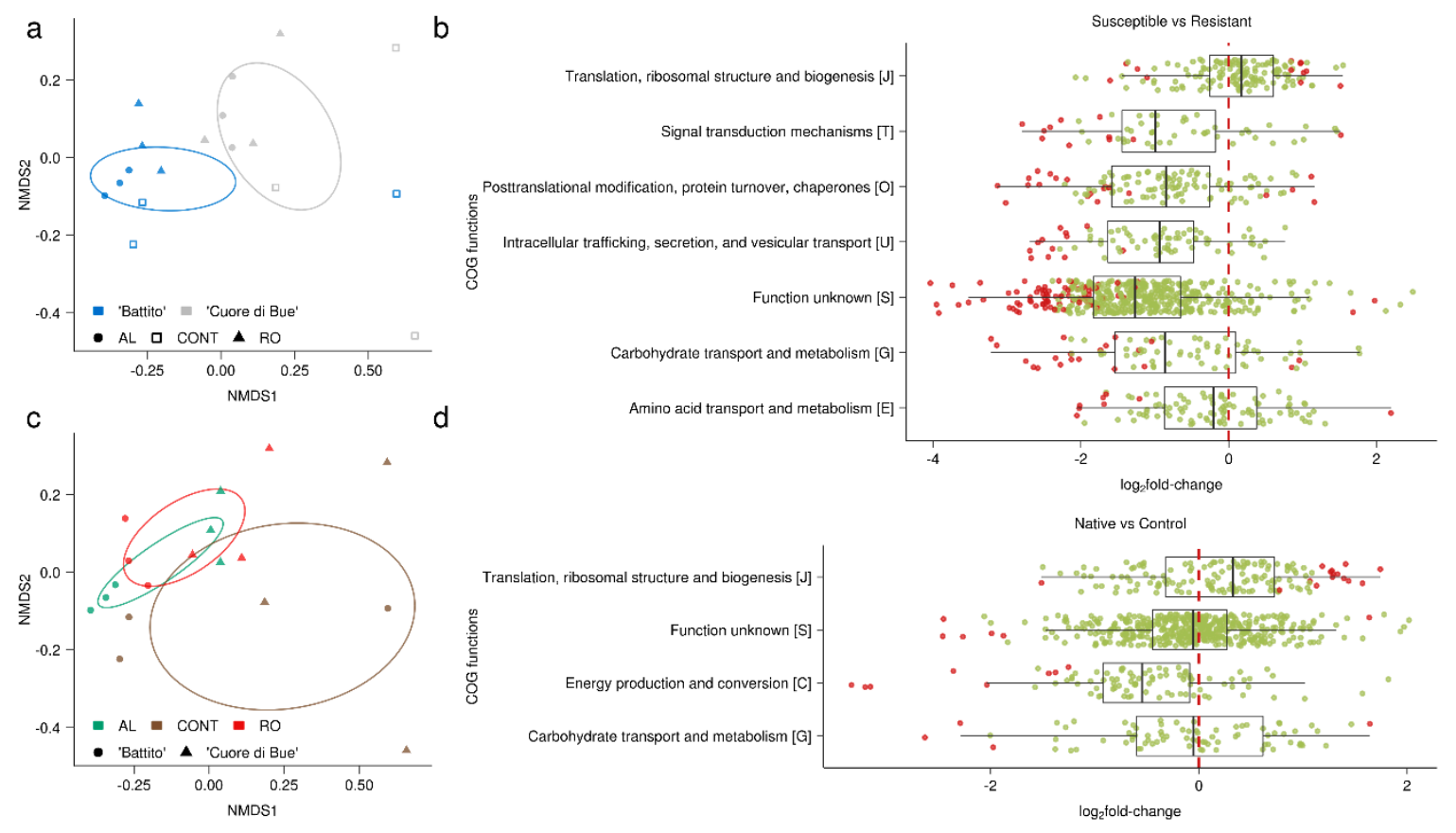

Figure 4. Meta-transcriptome functional diversity. (a,c) non-metric multidimensional scaling (NMDS) ordination plots on Bray-Curtis distances of expressed Clusters of Orthologous Groups (COG) functions clustered using 95\% confidence ellipses by genotype (a) and substrate type (c); stress =0.0776. AL, "Albenga" suppressive soil; RO, "Rosta" conducive soil; CONT, neutral control soil. (b,d) Boxplot of differentially expressed COG functions (FDR < 0.05) between genotypes (susceptible versus resistant, (b), and substrate type (native versus control soils, (d)). COGs are clustered by the more frequently represented functional categories, and points represent COG terms for each functional category; COGs that are significantly differentially expressed (FDR $<0.05)$ are indicated in red, COGs that are not in light green.

The functional diversity analysis revealed only a few basal metabolic functions, indicating that the sequencing depth was insufficient to capture a full profile of expressed microbial genes. However, we were able to detect major differences in the root-associated microbiota between native versus artificial substrates-both in the level of metabolic activity and in the genes expressed-and between different plant genotypes.

\subsection{Linking the Meta-Transcriptome with the Host Transcriptome}

Finally, we verified the feasibility of linking the taxonomic and functional diversity of the root-associated active microbiota, reconstructed from RNA-seq data, to the tomato transcriptome using variance-partitioning analysis. Meta-transcriptome features, namely LCA taxonomic assignments and functional diversity (expressed COG terms), were treated as explanatory variables of the variation in tomato expressed genes across conditions. We performed two independent analyses, first testing the influence of the taxonomic profile and then testing that of the functional profile (Figure 5). When considering the fungal and bacterial taxa associated with roots (Figure 5a), a large amount of plant transcriptome variance is explained by the active microbial diversity $(35 \%, p<0.001)$. By contrast, 
soil and genotype factors individually did not explain any of the transcriptome variance, although $2 \%$ of the explained variance was collinear to these two factors. Interestingly, $22 \%$ of the explained variance was collinear between soil type and microbial diversity, probably meaning that these two factors are interdependent in modulating tomato transcriptomic responses. Similarly, the genotype factor also shared $5 \%$ of collinearly explained variance with microbial diversity. The analysis was repeated considering active fungal and bacterial communities as independent factors. The bacterial community alone explained the greatest portion of plant transcriptome variance $(18 \%)$ with high collinearity with fungal diversity (Figure S2). The active fungal community, however, did not explain a significant portion of the variance on its own, being entirely collinear with other factors (mainly soil type and bacterial community).

a

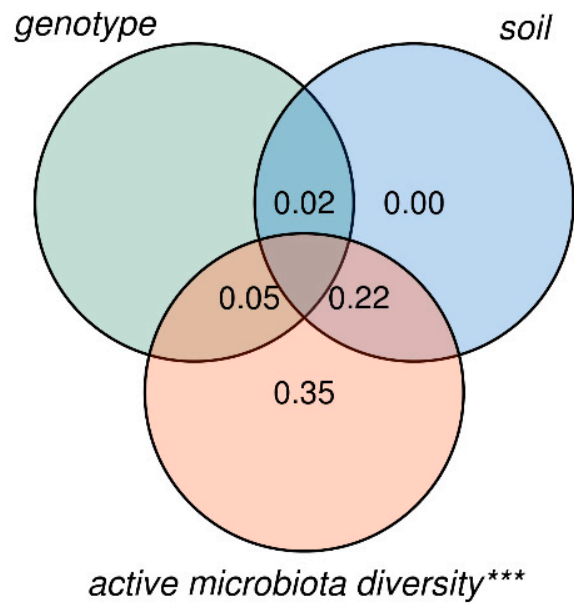

Residuals $=0.42$ b

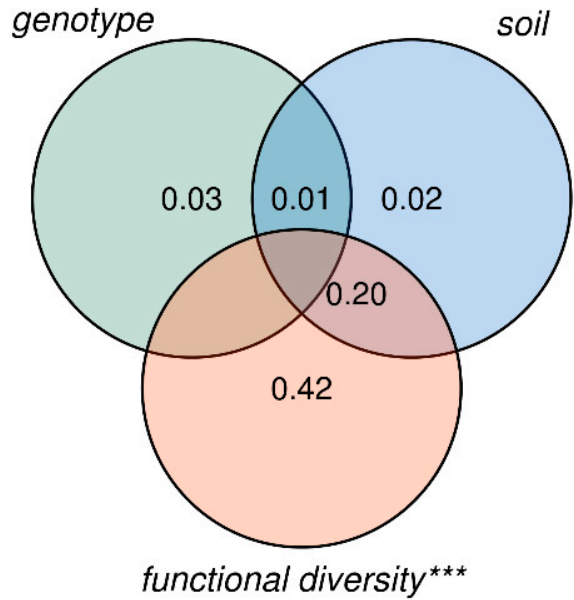

Residuals $=0.35$

Figure 5. Variance partitioning analysis on meta-transcriptome and plant transcriptome profiling. (a) Tomato transcriptome variance explained by genotype, soil, and active microbiota diversity (bacteria and fungi). (b) Tomato transcriptome variance explained by genotype, soil, and mapped COG terms (functional diversity). Residuals, unexplained variance fractions (discrepancy between the data and an estimation model). Significant fractions are indicated by asterisks (ANOVA on RDA model, ${ }^{*} p<0.05$; ** $\left.p<0.01 ;{ }^{* * *} p<0.001\right)$.

By using expressed COG functions within the meta-transcriptome as an explanatory variable, we were able to attribute $42 \%(p<0.001)$ of the plant transcriptome variance to microbial functions (Figure 5b). We also detected collinearity between soil type and COG terms that amounted to $20 \%$. However, unlike the results of the previous analysis, the variance explained exclusively by the soil factor dropped to $2 \%(p>0.05)$.

These results highlight that the taxonomic composition and in particular the expression profile of root-associated microbial communities correlate well with the plant expression profile across soils and genotypes, explaining more variance than genotype and soil factors. However, unexpectedly, the cumulative contribution of soil type and COGs in explaining tomato gene expression was slightly higher, similar to what we described above when considering meta-transcriptome diversity. This suggests that there is a strong relationship between soil features and meta-transcriptome functioning/diversity. 


\section{Conclusions}

Despite the low sequencing coverage of microbial transcripts, the deep mining of transcriptome datasets we previously generated from tomato plants grown in non-sterile environments [11] allowed the identification of a number of active microbes associated with roots. Our data demonstrate that this approach is feasible even with a high abundance of host sequences (>90\%) and a short read length (50 bp), even though the number of microbial reads (excluding rRNAs) harvested was considerably lower than was obtained in other meta-transcriptome studies $[57,58]$. Notwithstanding these technical constraints, we were able to reconstruct the active microbiota and functional diversity as reported in other works [43]. The reliability of the mined information was validated only for Glomeromycotina, so the accuracy and precision of our whole-community assemblage reconstructions need to be further confirmed, such as by generating simulated RNA-seq libraries at different read lengths and coverage values.

When considering the active diversity and functional profiles of the tomato-associated microbes, we found that the main driver of both is the host genotype, largely confirming previous data obtained in tomato [13] as well as in other plant models, such as rice [59], Arabidopsis [49], and Lotus [60]. However, especially in terms of taxonomic diversity, we also found a major impact of soil type, which was more conspicuous when considering the fungal communities. In addition to many bacteria, we detected arbuscular mycorrhizal fungi in the roots of both genotypes, but these fungi were more abundant in tomatoes growing in conducive RO soil than in suppressive AL soil, as was also confirmed by morphological quantification. At the same time, pathogenic fungi were less represented in AL soil, where bacteria belonging to Nocardiopsaceae and Kofleriaceae, known for their potential anti-microbial activity [52], were dominant. Taken as a whole, the data provided detailed information on the diversity of microbial communities associated with the roots of two tomato genotypes and supported our previous findings [11,12]. Lastly, through variance partitioning analysis we showed a good correlation between meta-transcriptome (including both taxonomic and functional diversity of microbes associated with roots) and plant host transcriptome.

In conclusion, the use of host-targeted RNA-seq libraries to study the meta-transcriptome is a feasible approach. It allows the reconstruction of microbial taxonomical and functional diversity at a relatively low sequencing depth (>90\% host sequences), maximizing the throughput of the RNA-seq approach in a cost-effective way.

Supplementary Materials: The following are available online at http://www.mdpi.com/2076-2607/8/1/38/s1, Figure S1: Work-flow diagram of the bioinformatic pipeline used for meta-transcriptome analysis; Figure S2: Global variance partitioning analysis of the plant transcriptome profiling explained by meta-transcriptome fungal and bacterial diversity. Table S1: Raw meta-transcriptome read counts alongside their lowest common ancestor (LCA) annotation (taxa identifiers refer to NCBI taxonomy) and differential abundance contrasts performed using DESeq2 R package (FDR < 0.05) for bacterial and fungal families. Table S2: Meta-transcriptome reads functional assignment on eggNOG 4.5 database and differential expression analysis performed using DESeq2 R package (FDR < 0.05).

Author Contributions: Conceptualization, M.C. and P.B.; methodology, M.C. and S.G.; formal analysis, M.C.; investigation, M.C. and M.N.; data curation, M.C.; writing-original draft preparation, M.C. and P.B.; writing-review and editing, M.C., P.B., S.G., M.N., W.N.H. and L.L.; visualization, M.C.; funding acquisition, P.B., W.N.H. and L.L. All authors have read and agreed to the published version of the manuscript.

Funding: This research was supported by the Mycoplant project (Root Microbiome for Plant Health: dissecting the role of soil fungi, TO_call03_2012_0039, Fondazione Compagnia di San Paolo Torino). This research has received funding from the European Union's Horizon 2020 research and innovation programme under grant agreement No 727929 (TOMRES). The authors are grateful to the Researchers Supporting Project number (RSP-2019/53), King Saud University, Riyadh, Saudi Arabia.

Acknowledgments: The authors thank Andrea Berruti for valuable help in multivariate statistics, Enrico Ercole, Paolo Bagnaresi, and Alessandra Salvioli for fruitful discussions. The authors are grateful to Dr. J. Mach for the critical reading and text editing of the manuscript.

Conflicts of Interest: The authors declare no conflict of interest. The funders had no role in the design of the study; in the collection, analyses, or interpretation of data; in the writing of the manuscript, or in the decision to publish the results. 


\section{References}

1. Cowan, D.A.; Ramond, J.-B.; Makhalanyane, T.P.; De Maayer, P. Metagenomics of extreme environments. Curr. Opin. Microbiol. 2015, 25, 97-102. [CrossRef] [PubMed]

2. Deng, Z.-L.; Gottschick, C.; Bhuju, S.; Masur, C.; Abels, C.; Wagner-Döbler, I. Metatranscriptome Analysis of the Vaginal Microbiota Reveals Potential Mechanisms for Protection against Metronidazole in Bacterial Vaginosis. Msphere 2018, 3, e00262-18. [CrossRef] [PubMed]

3. Crump, B.C.; Wojahn, J.M.; Tomas, F.; Mueller, R.S. Metatranscriptomics and Amplicon Sequencing Reveal Mutualisms in Seagrass Microbiomes. Front. Microbiol. 2018, 9, 388. [CrossRef] [PubMed]

4. Saminathan, T.; García, M.; Ghimire, B.; Lopez, C.; Bodunrin, A.; Nimmakayala, P.; Abburi, V.L.; Levi, A.; Balagurusamy, N.; Reddy, U.K. Metagenomic and Metatranscriptomic Analyses of Diverse Watermelon Cultivars Reveal the Role of Fruit Associated Microbiome in Carbohydrate Metabolism and Ripening of Mature Fruits. Front. Plant Sci. 2018, 9, 4. [CrossRef]

5. Cox, J.W.; Ballweg, R.A.; Taft, D.H.; Velayutham, P.; Haslam, D.B.; Porollo, A. A fast and robust protocol for metataxonomic analysis using RNAseq data. Microbiome 2017, 5, 7. [CrossRef]

6. Mateus, I.D.; Masclaux, F.G.; Aletti, C.; Rojas, E.C.; Savary, R.; Dupuis, C.; Sanders, I.R. Dual RNA-seq reveals large-scale non-conserved genotype $\times$ genotype-specific genetic reprograming and molecular crosstalk in the mycorrhizal symbiosis. ISME J. 2019, 13, 1226-1238. [CrossRef]

7. Kovalchuk, A.; Zeng, Z.; Ghimire, R.P.; Kivimäenpää, M.; Raffaello, T.; Liu, M.; Mukrimin, M.; Kasanen, R.; Sun, H.; Julkunen-Tiitto, R.; et al. Dual RNA-seq analysis provides new insights into interactions between Norway spruce and necrotrophic pathogen Heterobasidion annosum s.l. BMC Plant Biol. 2019, 19, 2. [CrossRef]

8. Young, E.; Carey, M.; Meharg, A.A.; Meharg, C. Microbiome and ecotypic adaption of Holcus lanatus (L.) to extremes of its soil $\mathrm{pH}$ range, investigated through transcriptome sequencing. Microbiome 2018, 6, 48. [CrossRef]

9. Celaj, A.; Markle, J.; Danska, J.; Parkinson, J. Comparison of assembly algorithms for improving rate of metatranscriptomic functional annotation. Microbiome 2014, 2, 39. [CrossRef]

10. Wood, D.E.; Salzberg, S.L. Kraken: Ultrafast metagenomic sequence classification using exact alignments. Genome Biol. 2014, 15, R46. [CrossRef]

11. Chialva, M.; Salvioli di Fossalunga, A.; Daghino, S.; Ghignone, S.; Bagnaresi, P.; Chiapello, M.; Novero, M.; Spadaro, D.; Perotto, S.; Bonfante, P. Native soils with their microbiotas elicit a state of alert in tomato plants. New Phytol. 2018, 220, 1296-1308. [CrossRef]

12. Chialva, M.; Zhou, Y.; Spadaro, D.; Bonfante, P. Not only priming: Soil microbiota may protect tomato from root pathogens. Plant Signal. Behav. 2018, 13, e1464855. [CrossRef] [PubMed]

13. Poli, A.; Lazzari, A.; Prigione, V.; Voyron, S.; Spadaro, D.; Varese, G.C. Influence of plant genotype on the cultivable fungi associated to tomato rhizosphere and roots in different soils. Fungal Biol. 2016, 120, 862-872. [CrossRef] [PubMed]

14. Voyron, S.; Ercole, E.; Ghignone, S.; Poli, A.; Spadaro, D.; Bonfante, P.; Girlanda, M. Plant Genotype Control over the Recruitment of the Tomato Fungal Microbiota. In Proceedings of the Ecology of Soil Microorganisms, Prague, Czech Republic, 29 November-3 December 2015; p. 175.

15. Leinonen, R.; Sugawara, H.; Shumway, M. International Nucleotide Sequence Database Collaboration The Sequence Read Archive. Nucleic Acids Res. 2011, 39, D19-D21. [CrossRef] [PubMed]

16. Sato, S.; Tabata, S.; Hirakawa, H.; Asamizu, E.; Shirasawa, K.; Isobe, S.; Kaneko, T.; Nakamura, Y.; Shibata, D.; Aoki, K.; et al. The tomato genome sequence provides insights into fleshy fruit evolution. Nature 2012, 485, 635-641.

17. Love, M.I.; Huber, W.; Anders, S. Moderated estimation of fold change and dispersion for RNA-seq data with DESeq2. Genome Biol. 2014, 15, 550. [CrossRef] [PubMed]

18. R Core Team. R: A Language and Environment for Statistical Computing; R Foundation for Statistical Computing: Vienna, Austria, 2019.

19. Bolger, A.M.; Lohse, M.; Usadel, B. Trimmomatic: A flexible trimmer for Illumina sequence data. Bioinformatics 2014, 30, 2114-2120. [CrossRef]

20. Dobin, A.; Davis, C.A.; Schlesinger, F.; Drenkow, J.; Zaleski, C.; Jha, S.; Batut, P.; Chaisson, M.; Gingeras, T.R. STAR: Ultrafast universal RNA-seq aligner. Bioinformatics 2013, 29, 15-21. [CrossRef] 
21. Langmead, B.; Salzberg, S.L. Fast gapped-read alignment with Bowtie 2. Nat. Methods 2012, 9, 357-359. [CrossRef]

22. Kopylova, E.; Noe, L.; Touzet, H. SortMeRNA: Fast and accurate filtering of ribosomal RNAs in metatranscriptomic data. Bioinformatics 2012, 28, 3211-3217. [CrossRef]

23. Database resources of the National Center for Biotechnology Information. Nucleic Acids Res. 2018, 46, D8-D13. [CrossRef] [PubMed]

24. Pongor, L.S.; Vera, R.; Ligeti, B. Fast and Sensitive Alignment of Microbial Whole Genome Sequencing Reads to Large Sequence Datasets on a Desktop PC: Application to Metagenomic Datasets and Pathogen Identification. PLoS ONE 2014, 9, e103441. [CrossRef] [PubMed]

25. Dick, J.M. Calculation of the relative metastabilities of proteins using the CHNOSZ software package. Geochem. Trans. 2008, 9, 10. [CrossRef] [PubMed]

26. Huerta-Cepas, J.; Szklarczyk, D.; Forslund, K.; Cook, H.; Heller, D.; Walter, M.C.; Rattei, T.; Mende, D.R.; Sunagawa, S.; Kuhn, M.; et al. eggNOG 4.5: A hierarchical orthology framework with improved functional annotations for eukaryotic, prokaryotic and viral sequences. Nucleic Acids Res. 2016, 44, D286-D293. [CrossRef] [PubMed]

27. Quinlan, A.R.; Hall, I.M. BEDTools: A flexible suite of utilities for comparing genomic features. Bioinformatics 2010, 26, 841-842. [CrossRef]

28. Buchfink, B.; Xie, C.; Huson, D.H. Fast and sensitive protein alignment using DIAMOND. Nat. Methods 2015, 12, 59-60. [CrossRef]

29. Trouvelot, A.; Kough, J.; Gianinazzi-Pearson, V. Mesure du taux de mycorhization VA d'un système radiculaire. Recherche de méthodes d'estimation ayant une signification fonctionnelle. In Physiological and Genetical Aspects of Mycorrhizae, Proceedings of the 1st European Symposium on Mycorrhizae, Dijon, France, 1-5 July 1985; Gianinazzi-Pearson, V., Gianinazzi, S., Eds.; Institut National de la Recherche Agronomique: Paris, France, 1986; pp. 217-221.

30. Oksanen, J.; Blanchet, F.G.; Friendly, M.; Kindt, R.; Legendre, P.; McGlinn, D.; Minchin, P.R.; O’Hara, R.B.; Simpson, G.L.; Solymos, P.; et al. Vegan: Community Ecology Package. R package Version 2.5-6. 2019. Available online: https://CRAN.R-project.org/package=vegan (accessed on 23 December 2019).

31. McMurdie, P.J.; Holmes, S. Waste Not, Want Not: Why Rarefying Microbiome Data Is Inadmissible. PLoS Comput. Biol. 2014, 10, e1003531. [CrossRef]

32. Yu, G.; Wang, L.-G.; Han, Y.; He, Q.-Y. clusterProfiler: An R package for comparing biological themes among gene clusters. OMICS 2012, 16, 284-287. [CrossRef]

33. Fox, J.; Weisberg, S. An R Companion to Applied Regression, 3rd eds.; Sage: Thousand Oaks, CA, USA, 2019.

34. Levene, H. Robust tests for equality of variances. In Contributions to Probability and Statistics: Essays in Honor of Harold Hotelling; Olkin, I., Ed.; Stanford University Press: Palo Alto, CA, USA, 1960; pp. 278-292.

35. Kruskal, W.H. A Nonparametric test for the Several Sample Problem. Ann. Math. Stat. 1952, 23, 525-540. [CrossRef]

36. Tukey, J.W. Comparing Individual Means in the Analysis of Variance. Biometrics 1949, 5, 99-114. [CrossRef]

37. De Mendiburu, F. Agricolae: Statistical Procedures for Agricultural Research. R Package Version 1.3-1. 2019. Available online: https:/CRAN.R-project.org/package=agricolae (accessed on 23 December 2019).

38. Dunn, O.J. Multiple Comparisons Using Rank Sums. Technometrics 1964, 6, 241-252. [CrossRef]

39. Ogle, D.H.; Wheeler, P.; Dinno, A. FSA: Fisheries Stock Analysis. R Package Version 0.8.26. 2019. Available online: https://github.com/droglenc/FSA (accessed on 23 December 2019).

40. Derksen, S.; Keselman, H.J. Backward, forward and stepwise automated subset selection algorithms: Frequency of obtaining authentic and noise variables. Br. J. Math. Stat. Psychol. 1992, 45, 265-282. [CrossRef]

41. Dray, S.; Blanchet, G.; Borcard, D.; Guenard, G.; Jombart, T.; Larocque, G.; Legendre, P.; Madi, N.; Wagner, H.H. Adespatial: Multivariate Multiscale Spatial Analysis. R package Version 0.3-7. 2019. Available online: https://CRAN.R-project.org/package=adespatial (accessed on 23 December 2019).

42. Xiong, X.; Frank, D.N.; Robertson, C.E.; Hung, S.S.; Markle, J.; Canty, A.J.; McCoy, K.D.; Macpherson, A.J.; Poussier, P.; Danska, J.S.; et al. Generation and Analysis of a Mouse Intestinal Metatranscriptome through Illumina Based RNA-Sequencing. PLoS ONE 2012, 7, e36009. [CrossRef] [PubMed]

43. Westreich, S.T.; Korf, I.; Mills, D.A.; Lemay, D.G. SAMSA: A comprehensive metatranscriptome analysis pipeline. BMC Bioinform. 2016, 17, 399. [CrossRef] [PubMed] 
44. Danielsen, L.; Thürmer, A.; Meinicke, P.; Buée, M.; Morin, E.; Martin, F.; Pilate, G.; Daniel, R.; Polle, A.; Reich, M. Fungal soil communities in a young transgenic poplar plantation form a rich reservoir for fungal root communities. Ecol. Evol. 2012, 2, 1935-1948. [CrossRef]

45. Palaniyandi, S.A.; Yang, S.H.; Zhang, L.; Suh, J.-W. Effects of actinobacteria on plant disease suppression and growth promotion. Appl. Microbiol. Biotechnol. 2013, 97, 9621-9636. [CrossRef]

46. Poomthongdee, N.; Duangmal, K.; Pathom-aree, W. Acidophilic actinomycetes from rhizosphere soil: Diversity and properties beneficial to plants. J. Antibiot. 2015, 68, 106-114. [CrossRef]

47. Detheridge, A.P.; Brand, G.; Fychan, R.; Crotty, F.V.; Sanderson, R.; Griffith, G.W.; Marley, C.L. The legacy effect of cover crops on soil fungal populations in a cereal rotation. Agric. Ecosyst. Environ. 2016, 228, $49-61$. [CrossRef]

48. Bulgarelli, D.; Rott, M.; Schlaeppi, K.; Ver Loren van Themaat, E.; Ahmadinejad, N.; Assenza, F.; Rauf, P.; Huettel, B.; Reinhardt, R.; Schmelzer, E.; et al. Revealing structure and assembly cues for Arabidopsis root-inhabiting bacterial microbiota. Nature 2012, 488, 91-95. [CrossRef]

49. Lundberg, D.S.; Lebeis, S.L.; Paredes, S.H.; Yourstone, S.; Gehring, J.; Malfatti, S.; Tremblay, J.; Engelbrektson, A.; Kunin, V.; Del Rio, T.G.; et al. Defining the core Arabidopsis thaliana root microbiome. Nature 2012, 488, 86-90. [CrossRef]

50. Alabouvette, C.; Olivain, C.; L-Haridon, F.; Aimé, S.; Steinberg, C. Using strains of Fusarium oxysporum to control Fusarium wilts: Dream or reality? In Novel Biotechnologies for Biocontrol Agent Enhancement and Management; Vurro, M., Gressel, J., Eds.; NATO Security through Science Series; Springer: Dordrecht, The Netherlands, 2007; pp. 157-177, ISBN 978-1-4020-5799-1.

51. Green, S.J.; Inbar, E.; Michel, F.C.; Hadar, Y.; Minz, D. Succession of Bacterial Communities during Early Plant Development: Transition from Seed to Root and Effect of Compost Amendment. Appl. Environ. Microbiol. 2006, 72, 3975-3983. [CrossRef] [PubMed]

52. Mendes, R.; Kruijt, M.; De Bruijn, I.; Dekkers, E.; Van der Voort, M.; Schneider, J.H.M.; Piceno, Y.M.; DeSantis, T.Z.; Andersen, G.L.; Bakker, P.A.H.M.; et al. Deciphering the Rhizosphere Microbiome for Disease-Suppressive Bacteria. Science 2011, 332, 1097-1100. [CrossRef] [PubMed]

53. Rosendahl, S. Communities, populations and individuals of arbuscular mycorrhizal fungi. New Phytol. 2008, 178, 253-266. [CrossRef] [PubMed]

54. Öpik, M.; Moora, M.; Liira, J.; Zobel, M. Composition of root-colonizing arbuscular mycorrhizal fungal communities in different ecosystems around the globe. J. Ecol. 2006, 94, 778-790. [CrossRef]

55. Teixeira, P.J.P.; Colaianni, N.R.; Fitzpatrick, C.R.; Dangl, J.L. Beyond pathogens: Microbiota interactions with the plant immune system. Curr. Opin. Microbiol. 2019, 49, 7-17. [CrossRef]

56. Del Giorgio, P.A.; Cole, J.J. Bacterial Growth Efficiency in Natural Aquatic Systems. Annu. Rev. Ecol. Syst. 1998, 29, 503-541. [CrossRef]

57. Leimena, M.M.; Ramiro-Garcia, J.; Davids, M.; Van den Bogert, B.; Smidt, H.; Smid, E.J.; Boekhorst, J.; Zoetendal, E.G.; Schaap, P.J.; Kleerebezem, M. A comprehensive metatranscriptome analysis pipeline and its validation using human small intestine microbiota datasets. BMC Genom. 2013, 14, 530. [CrossRef]

58. Liao, W.; Ren, J.; Wang, K.; Wang, S.; Zeng, F.; Wang, Y.; Sun, F. Alignment-free Transcriptomic and Metatranscriptomic Comparison Using Sequencing Signatures with Variable Length Markov Chains. Sci. Rep. 2016, 6, 1-15. [CrossRef]

59. Zhang, J.; Liu, Y.-X.; Zhang, N.; Hu, B.; Jin, T.; Xu, H.; Qin, Y.; Yan, P.; Zhang, X.; Guo, X.; et al. NRT1.1B is associated with root microbiota composition and nitrogen use in field-grown rice. Nat. Biotechnol. 2019, 37, 676-684. [CrossRef]

60. Zgadzaj, R.; Garrido-Oter, R.; Jensen, D.B.; Koprivova, A.; Schulze-Lefert, P.; Radutoiu, S. Root nodule symbiosis in Lotus japonicus drives the establishment of distinctive rhizosphere, root, and nodule bacterial communities. Proc. Natl. Acad. Sci. USA 2016, 113, E7996-E8005. [CrossRef]

(C) 2019 by the authors. Licensee MDPI, Basel, Switzerland. This article is an open access article distributed under the terms and conditions of the Creative Commons Attribution (CC BY) license (http://creativecommons.org/licenses/by/4.0/). 